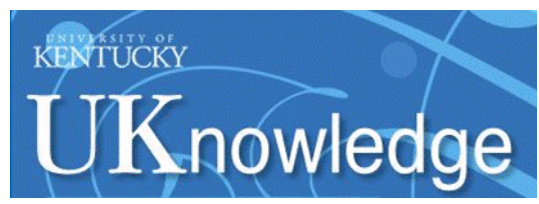

University of Kentucky

UKnowledge

\title{
Gemini Mid-IR Polarimetry of NGC 1068: Polarized Structures around the Nucleus
}

\author{
C. Packham \\ University of Florida
}

S. Young

University of Hertfordshire, United Kingdom

S. Fisher

Northern Operations Center

K. Volk

Northern Operations Center

R. Mason

Northern Operations Center

See next page for additional authors

Follow this and additional works at: https://uknowledge.uky.edu/physastron_facpub

Part of the Astrophysics and Astronomy Commons, and the Physics Commons

Right click to open a feedback form in a new tab to let us know how this document benefits you.

\section{Repository Citation}

Packham, C.; Young, S.; Fisher, S.; Volk, K.; Mason, R.; Hough, J. H.; Roche, P. F.; Elitzur, Moshe; Radomski, J.; and Perlman, E., "Gemini Mid-IR Polarimetry of NGC 1068: Polarized Structures around the Nucleus" (2007). Physics and Astronomy Faculty Publications. 205.

https://uknowledge.uky.edu/physastron_facpub/205

This Article is brought to you for free and open access by the Physics and Astronomy at UKnowledge. It has been accepted for inclusion in Physics and Astronomy Faculty Publications by an authorized administrator of UKnowledge. For more information, please contact UKnowledge@lsv.uky.edu. 
Gemini Mid-IR Polarimetry of NGC 1068: Polarized Structures around the Nucleus

Digital Object Identifier (DOI)

http://dx.doi.org/10.1086/518602

Notes/Citation Information

Published in The Astrophysical Journal Letters, v. 661, no. 1, p. L29-L32.

(c) 2007. The American Astronomical Society. All rights reserved.

The copyright holder has granted permission for posting the article here.

Authors

C. Packham, S. Young, S. Fisher, K. Volk, R. Mason, J. H. Hough, P. F. Roche, Moshe Elitzur, J. Radomski, and E. Perlman

This article is available at UKnowledge: https://uknowledge.uky.edu/physastron_facpub/205 


\title{
GEMINI MID-IR POLARIMETRY OF NGC 1068: POLARIZED STRUCTURES AROUND THE NUCLEUS
}

\author{
C. Packham, ${ }^{1}$ S. Young,${ }^{2}$ S. Fisher, ${ }^{3}$ K. Volk,${ }^{3}$ R. Mason,${ }^{3}$ J. H. Hough, ${ }^{2}$ \\ P. F. Roche, ${ }^{4}$ M. Elitzur, ${ }^{5}$ J. Radomski, ${ }^{6}$ and E. Perlman ${ }^{7}$ \\ Received 2007 February 23; accepted 2007 April 3; published 2007 May 3
}

\begin{abstract}
We present diffraction-limited, $10 \mu \mathrm{m}$ imaging polarimetry data for the central regions of the archetypal Seyfert active galactic nucleus NGC 1068. The position angle of polarization is consistent with three dominant polarizing mechanisms. We identify three distinct regions of polarization: (1) north of the nucleus, arising from aligned dust in the narrow emission line region, (2) south, east, and west of the nucleus, consistent with dust being channeled toward the central engine, and (3) a central minimum of polarization consistent with a compact $(\leq 22 \mathrm{pc})$ torus. These observations provide continuity between the geometrically and optically thick torus and the host galaxy's nuclear environments. These images represent the first published mid-IR polarimetry from an 8 m-class telescope and illustrate the potential of such observations.
\end{abstract}

Subject headings: galaxies: active — galaxies: nuclei — galaxies: Seyfert — infrared: galaxies — instrumentation: polarimeters - polarization

Online material: color figure

\section{INTRODUCTION}

The unified theory of Seyfert-type active galactic nuclei (AGNs) holds that all types of Seyfert AGNs are essentially the same object, viewed from different lines of sight. Surrounding the central engine is a geometrically and optically thick, dusty, molecular torus, obscuring the broad emission line region from some lines of sight. In this scheme, the Seyfert classification depends solely on the lines of sight and on the exact torus properties. Such theories received a major boost through the detection of scattered, and hence polarized, broad emission lines in the spectrum of NGC 1068 (Antonucci \& Miller 1985), revealing an obscured Seyfert 1 central engine in the previously classified Seyfert 2 AGN, entirely consistent with unified theories.

While fundamental to unified theories, the torus remains difficult to image directly at optical/IR wavelengths, with perhaps the most direct observation of the torus made by speckle interferometry in the near-IR (Weigelt et al. 2004). Strong evidence for significant amounts of obscuring material in the central 100 pc-scale nuclear regions of NGC 1068, possibly in the form of a torus, is provided by observations of $\mathrm{CO}$ and HCN emission (Planesas et al. 1991; Jackson et al. 1993; Schinnerer et al. 2000) and recent Chandra X-ray observations (e.g., Ogle et al. 2003). Mid-IR spectroscopy reveals a moderately deep $\left(\tau_{9.7} \approx 0.4\right)$ silicate absorption feature at the nucleus (Roche et al. 1984; Siebenmorgen et al. 2004), whose strength is approximately constant up to $\sim 1$ " south of the brightest midIR point (Mason et al. 2006; Rhee \& Larkin 2006). Applying

\footnotetext{
${ }^{1}$ University of Florida, Department of Astronomy, 211 Bryant Space Science Center, P.O. Box 112055, Gainesville, FL 32611-2055.

${ }^{2}$ Center for Astrophysics Research, University of Hertfordshire, Hatfield, Hertfordshire AL10 9AB, UK.

${ }^{3}$ Gemini Observatory, Northern Operations Center, 670 North A'ohuku Place, Hilo, HI 96720.

${ }^{4}$ University of Oxford, Department of Astrophysics, Keble Road, Oxford OX1 3RH, UK

${ }^{5}$ University of Kentucky, Department of Physics and Astronomy, 600 Rose Street, Lexington, KY 40506.

${ }^{6}$ Gemini Observatory, Southern Operations Center, c/o AURA, Inc., Casilla 603, La Serena, Chile.

${ }^{7}$ Physics and Space Sciences Department, Florida Institute of Technology, 150 West University Boulevard, Melbourne, FL 32901-6988.
}

the Nenkova et al. (2002) clumpy torus model, Mason et al. (2006) suggested that the torus is compact $(\leq 15 \mathrm{pc})$, in good agreement with mid-IR interferometric observations (Jaffe et al. 2004). Further evidence for a compact torus was found through adaptive optics-fed $\mathrm{H}_{2} 1-0 S(1)$ observations by Davies et al. (2006), who found a 15 pc clump of $\mathrm{H}_{2}$ extending from the nucleus at the same position angle as the line of masers. The observed extent of the torus, or nuclear obscuring material in NGC 1068, is partly dependent on the wavelength and/or observational technique. Young et al. (1996) used imaging polarimetry to observe the silhouette of obscuring material against the southern ionization cone, which they attributed to the torus, with a derived diameter of $\sim 200 \mathrm{pc}$ in the $H$ band.

The close proximity $\left(1^{\prime \prime}=72 \mathrm{pc}\right)$ and high brightness of NGC 1068 make it an ideal target for polarimetry, a traditionally photon-starved application. Near-IR studies of NGC 1068 by Packham et al. (1997), Lumsden et al. (1999), and Simpson et al. (2002) clearly detected the biconical ionization structure in scattered light. In the nuclear regions, there is a trend to a position angle (P.A.) of polarization being perpendicular to the radio jet with increasing wavelength. Modeling of the nuclear regions requires both an extended area of scattering particles as well as dichroic absorption of nuclear emission, possibly by dust in, or associated with, the torus (Young et al. 1995; Watanabe et al. 2003).

Bailey et al. (1988) found that the P.A. of polarization rotates by $\sim 70^{\circ}$ between 4 and $5 \mu \mathrm{m}$. The $10 \mu \mathrm{m}$ spectropolarimetry of Aitken et al. (1984) showed a similar P.A. of polarization to that at $5 \mu \mathrm{m}$, and a constant degree of polarization through the silicate absorption feature. These results are entirely consistent with the predicted $90^{\circ}$ change from dichroic absorption to dichroic emission from aligned dust grains. That the P.A. change was only $\sim 70^{\circ}$ is attributable to dilution of the dichroic emission component by polarized flux in the extended scattering cones, most likely from dichroic emission from dust in the narrow emission line region (Bailey et al. 1988).

To investigate the contributions of the various polarizing mechanisms and structures in the nucleus of NGC 1068, Lumsden et al. (1999) performed imaging polarimetry using a broadband $8-13 \mu \mathrm{m}$ filter. These data represented the first and only published mid-IR imaging polarimetry of an AGN, but their 


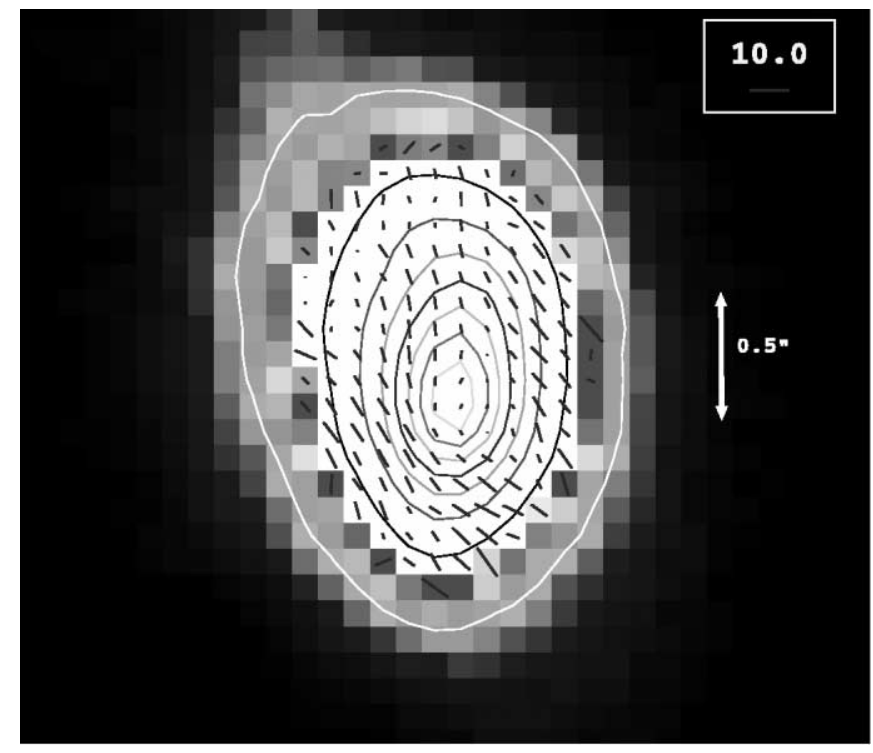

FIG. 1.-Total flux image (color) with the polarization vectors for the central regions of NGC 1068. The length of the vector is proportional to the degree of polarization, and the angle shows the P.A. of polarization. Each pixel is $0.1^{\prime \prime}$, and the $10 \%$ polarization scale bar is shown in the upper right. North is up, and east is to the left. [See the electronic edition of the Journal for a color version of this figure.]

interpretation was complicated by the $\sim 0.7^{\prime \prime}$ resolution of the data. To take advantage of the improved spatial resolution attainable from an $8 \mathrm{~m}$-class telescope, we obtained new midIR imaging polarimetry of NGC 1068 during commissioning of this mode at the Gemini North telescope.

\section{OBSERVATIONS}

We obtained imaging polarimetry of NGC 1068 during commissioning of the polarimetry unit of Michelle (Glasse et al. 1997) on UT 2005 December 19 on the Gemini North $8.1 \mathrm{~m}$ telescope. These observations were primarily aimed at measuring the degree and position angle of polarization with NGC 1068 as a test object, and hence used a limited on-source time of 148 s. Michelle uses a Raytheon $320 \times 240$ pixel Si:As IBC array, providing a plate scale of $0.1^{\prime \prime}$ pixel $^{-1}$ in imaging mode. Images were obtained in the $9.7 \mu \mathrm{m}(\delta \lambda=1.0 \mu \mathrm{m}$, $50 \%$ cut-on/off) filter only, using the standard chop-nod technique to remove time-variable sky background, telescope thermal emission, and so-called 1/f detector noise. The chop throw was $15^{\prime \prime}$, and the telescope was nodded every $\sim 90 \mathrm{~s}$. The chop throw was fixed at $0^{\circ}$ (north-south). Conditions were photometric, and the observations were diffraction-limited $\left(\sim 0.30^{\prime \prime}\right.$ FWHM).

Michelle employs a warm, rotatable half-wave retarder (or half-wave plate, HWP) to modulate the polarization signal, located upstream of the entrance window of the dewar. A cold wire grid polarizer is used as the polarimetric analyzer, located in a collimated beam. Images were taken at four HWP P.A.'s in the following sequence: $0^{\circ}, 45^{\circ}, 45^{\circ}, 0^{\circ}, 22.5^{\circ}, 67.5^{\circ}, 67.5^{\circ}$, $22.5^{\circ}$ in the first nod position, and the sequence repeated in the second nod position. In this manner, the Stokes parameters can be computed as close in time as possible, reducing the effects of variations in sky transmission and emission. This sequence, however, requires many motions of the HWP and is therefore under evaluation with a view to reducing the number of HWP motions to increase observing efficiency. Data were reduced using the Gemini IRAF package in conjunction with

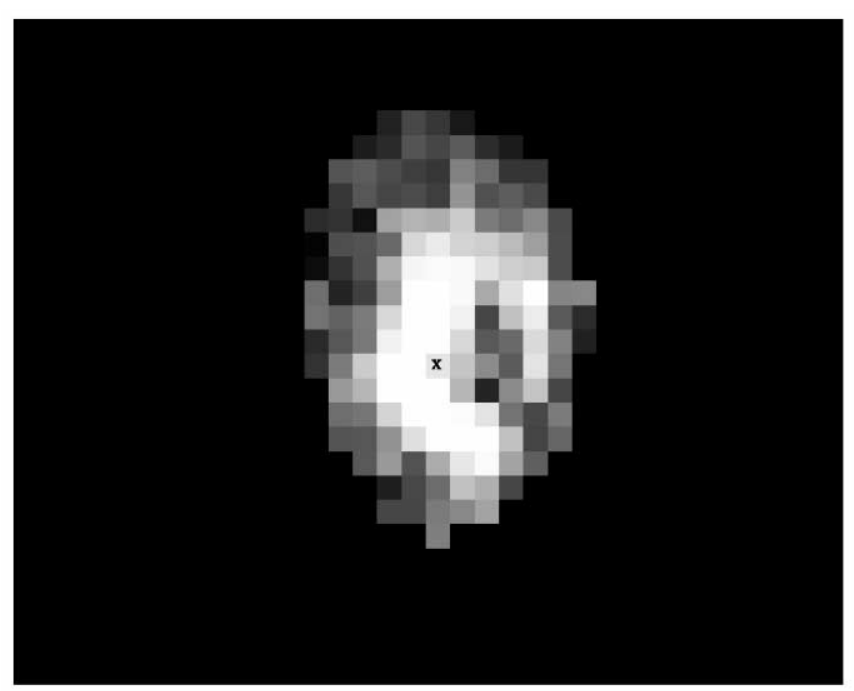

FIG. 2.-Polarized flux image of the central regions of NGC 1068. The " $\mathrm{X}$ " shows the position of the peak total flux. Each pixel is $0.1^{\prime \prime}$.

Starlink POLPACK software (Berry \& Gledhill 2003). The difference for each chop pair in a given nod position and HWP P.A. was calculated, and then differenced with the second nod position at the same HWP P.A. Images were aligned through shifting by fractional pixel values to account for slight image drift between frames, and then the Stokes parameters $I, Q$, and $U$ computed using POLPACK. A total of 20 nod positions were recorded, and residual array/electronic noise was removed through use of a median-filter noise mask. The data were reduced through the creation of four individual $I, Q$, and $U$ maps and also through co-adding all frames at their respective HWP P.A.'s first and then producing a single $I, Q$, and $U$ map. The signal-to-noise ratio $(\mathrm{S} / \mathrm{N})$ in the latter method is slightly higher in the individual $Q$ and $U$ maps, presumably due to a "smoothing" of the $Q$ and $U$ during the co-addition; these are the data used in this Letter.

The efficiency and zero-angle calibration of the polarimeter were measured through observations of two polarized sources, and the comparison with measurements published by Smith et al. (2000). The instrumental polarization was estimated to be $\leq 0.3 \%$ through observations of two stars that fulfilled the criteria of (1) high proper motion (hence nearby), (2) high Galactic latitude (to minimize the presence of Galactic dust), and (3) an intermediate spectral-type star (to minimize intrinsic stellar nebulosity).

\section{RESULTS}

Figure 1 shows the total flux image (color scale and contours) with the polarization vectors overlaid. The polarization vectors are plotted where the $\mathrm{S} / \mathrm{N}$ is $\geq 54$ in total flux, and contours are linearly spaced in intensity, starting at a $\mathrm{S} / \mathrm{N}$ of 27. Figure 2 shows the polarized intensity map, produced by multiplying the degree of polarization by the total intensity image. As in Figure 1, only where the $\mathrm{S} / \mathrm{N}$ in the total intensity image is $\geq 54$ are data plotted. The resultant polarization vectors are contained within an approximate north-south oriented ellipse, major/minor axes $1.7^{\prime \prime} / 1.2^{\prime \prime}$, respectively. The integrated degree of polarization within that ellipse is $2.48 \% \pm 0.57 \%$ at a P.A. of $26.7^{\circ} \pm 15.3^{\circ}$. The errors in the degree and P.A. of polarization are estimated through independent measurements of the four individual polarization maps and by computing the standard deviation. It should be noted that the ex- 
TABLE 1

Polarization Component Summary

\begin{tabular}{|c|c|c|c|c|c|}
\hline Locale & $\begin{array}{l}\text { Aperture } \\
(\operatorname{arcsec})\end{array}$ & $\begin{array}{c}\lambda \\
(\mu \mathrm{m})\end{array}$ & $\begin{array}{l}\text { Degree of Polarization } \\
\qquad(\%)\end{array}$ & $\begin{array}{l}\text { P.A. of Polarization } \\
\text { (deg) }\end{array}$ & Emitting Component \\
\hline 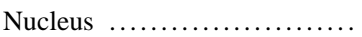 & $1.7 \times 1.2$ & $9.7^{\mathrm{a}}$ & $2.48 \pm 0.57$ & $26.7 \pm 15.3$ & Several \\
\hline North region $\ldots \ldots \ldots \ldots \ldots \ldots$ & $\ldots$ & $9.7^{\mathrm{a}}$ & $\sim 2$ & $\sim 8$ & Dichroic emission from dust aligned through jet interaction \\
\hline East, west, south regions ....... & $\ldots$ & $9.7^{\mathrm{a}}$ & $\sim 3.5$ & $\sim 35$ & Dichroic emission from galactic dust or torus outer edge \\
\hline Innermost region $\ldots \ldots \ldots \ldots \ldots$ & $\ldots$ & $9.7^{\mathrm{a}}$ & $\leq 1$ & $\ldots$ & Dichroic absorption or unpolarized source \\
\hline 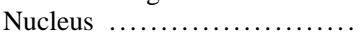 & 2 & $10^{\mathrm{b}}$ & $1.3 \pm 0.05$ & $49 \pm 3$ & Several, including dichroic emission \\
\hline & 2 & $2.2^{\mathrm{c}}$ & $4.11 \pm 0.46$ & $120.6 \pm 2.38$ & Several, including dichroic absorption \\
\hline
\end{tabular}

${ }^{a}$ The $9.7 \mu \mathrm{m}$ data from this Letter.

${ }^{\mathrm{b}}$ The $10 \mu \mathrm{m}$ data from Lumsden et al. (1999).

${ }^{\mathrm{c}}$ The $2.2 \mu \mathrm{m}$ data from Packham et al. (1997).

posure time in those four individual maps was very short, where systematic effects could dominate, and hence the quoted errors may be an overestimation. The degree of polarization is higher than the $1.30 \%$ measured by Lumsden et al. (1999) in a $2^{\prime \prime}$ aperture, consistent with an increased observed polarization as often arises with improved spatial resolution. The P.A. of polarization is significantly different from Lumsden et al.'s measurement of $49^{\circ}$ in a $2^{\prime \prime}$ aperture. However, our data shows a P.A. rotation of $94^{\circ}$ from the $K_{n}$-band data of Packham et al. (1997) and Lumsden et al. (1999), entirely as expected if the dominant polarizing mechanism changes from dichroic absorption to emission between the two wave bands, as described in $\S 1$. We speculate that the Lumsden et al. (1999) $\sim 0.68^{\prime \prime}$ results suffered significantly greater contamination in their beam, possibly from surrounding extended polarization, as compared to our $\sim 0.30^{\prime \prime}$ results. In addition, the wider bandwidth of Lumsden et al. (1999) would have been more affected by the different and competing polarizing components.

The degree and P.A. of polarization suggest contributions from three components. The first extends $\sim 1$ " north of the midIR peak and is coincident with the inner regions of the radio jet, with a P.A. of polarization approximately north-south. The second region extends south, east, and west of the nucleus, with a P.A. of polarization of $\sim 35^{\circ}$. Finally, the degree of polarization drops to a minimum very close to the mid-IR total flux peak, which we believe is most likely to arise from an unresolved polarization contribution with a P.A. of polarization approximately orthogonal to that of the more extended emission, leading to a reduction in the measured polarization. The polarized intensity image reveals polarized emission extending north of the mid-IR peak, two areas of enhanced flux east and west of the mid-IR peak, and a minimum close to the mid-IR total flux peak. Table 1 summarizes the locations and polarization components.

\section{DISCUSSION}

Polarization at mid-IR wavelengths most likely arises from either dichroic absorption or dichroic emission, both due to dust grains with a preferred alignment. The integrated P.A. of polarization in these data confirms and enhances the interpretation of the near $90^{\circ}$ P.A. flip from near- to mid-IR wavelengths, with the factor $\sim 2.5$ increase in spatial resolution providing a more accurate and consistent result. Galliano et al. (2003, 2005) suggested, based on spatial coincidence, that the [O III] clouds in the ionization cone are the dominant mid-IR sources away from the compact torus. The polarized flux image shows a similar spatial correspondence with the [O III], and the P.A. of polarization north of the nucleus is consistent with the interpretation of Bailey et al. (1988) of dichroic emission in the narrow-line region, possibly through dust alignment via jet streaming or a helical magnetic field associated with the jet. We discount directly observed synchrotron radiation from the radio jet accounting for the polarization, as an extrapolation of the radio emission to the mid-IR provides too little flux. Hence, these data confirm that the extended mid-IR polarized emission north of the nucleus is mostly from dust in the ionization cone.

South, east, and west of the nucleus, as the P.A. of polarization is perpendicular to that in the near-IR where the polarization is thought to be produced by dichroic absorption, we suggest that the dominant polarizing mechanism is dichroic emission by grains aligned to the same field direction as the absorbing grains, in agreement with other authors (i.e., Bailey et al. 1988). Due to the Barnett effect (Lazarian 2003 and references therein), grains align with their short axes parallel to the local magnetic field, and the P.A. of polarization is parallel to the direction of the magnetic field for dichroic absorption and orthogonal for dichroic emission. The location of the polarized emission areas and the P.A. of polarization is suggestive of warm aligned dust grains being channeled from the host galaxy toward the torus. Indeed, the P.A. of the polarized flux is coincident with the $\mathrm{H}_{2}$ material that Davies et al. (2006) associated with molecular material in a compact torus.

Dichroic absorption in an unresolved optically thick central region could account for the minimum in polarization close to the peak of mid-IR flux, with a P.A. of polarization approximately orthogonal to the more extended dichroic emission to the east, west, and south. Alternatively, the mid-IR flux in the innermost regions could arise from a strong mid-IR, intrinsically unpolarized, source. However, there is tentative evidence of the central regions showing a twist in the P.A. of polarization, tending toward a similar P.A. found in the dichroic absorption at near-IR wavelengths (e.g., Packham et al. 1997), which supports the dichroic absorption interpretation. In both possibilities, a compact $\left(\leq 0.3^{\prime \prime}[\leq 22 \mathrm{pc}]\right.$ diameter) torus could account for this result. If correct, the polarization minimum indicates the true position of the central engine, which is not coincident with the mid-IR total flux peak but is displaced by $\sim 0.2^{\prime \prime}$ to the west.

CO (Schinnerer et al. 2000) and optical Hubble Space Telescope (Catchpole \& Boksenberg 1997) observations are interpreted as evidence of a warped molecular disk on $100 \mathrm{pc}$ scales, partially obscuring the nuclear regions of the host galaxy and ionization cone pointing away from Earth. Indeed, Schinnerer et al. (2000) speculate that this material, rather than a compact torus, is responsible for obscuring the AGN. Elitzur \& Shlosman (2006) suggest that the 100 pc molecular structure is the extension of the parsec-scale disk of masers (Greenhill \& Gwinn 1997; Gallimore et al. (2001; Galliano et al. 2003). We suggest that our data provide continuity between the geomet- 
rically thick torus (height/radius $\sim 1$ ) and the flatter, larger galactic disk (height/radius $\sim 0.15$ ).

The western polarized feature is considerably larger than the compact (less than or equal to a few parsecs) torus suggested by several authors (e.g., Jaffe et al. 2004; Mason et al. 2006; Packham et al. 2005; Radomski et al. 2006) on the basis of mid-IR imaging and modeling, but much smaller than the suggested torus detected by Young et al. (1996). However, the Young et al. (1996) feature is detected in polarized flux, a technique that increases contrast by removing the dominant, unpolarized, emission. Distinct from total flux, polarimetric observations are therefore potentially much more sensitive to emission from the putative faint, outer regions of the torus where the interaction with the inner regions of the host galaxy must occur. We suggest that a way to reconcile the evidence for a compact torus with these observations and others, such as extended silicate absorption (Roche et al. 2006, 2007) and $100 \mathrm{pc}-$ scale CO disks, is that the compact, geometrically, and optically thick torus is often surrounded by a larger, more diffuse structure, associated with the dusty central regions of the host galaxy. Where the torus ends and the host galaxy dust structure starts may be more of a question of semantics rather than a true physical boundary. These observations examine the interaction between the host galaxy and possible entrainment into the outer torus regions. Further multiple-wavelength polarimetric observations of both NGC 1068 and other AGNs are required to test this hypothesis.

We are grateful to the Gemini, UKIRT, and ATC science and engineering staff for their outstanding work on Michelle and the Gemini telescope, and wish to note especially Chris Carter. This work is based on observations obtained at the Gemini Observatory, which is operated by the Association of Universities for Research in Astronomy, Inc., under a cooperative agreement with the NSF on behalf of the Gemini partnership: the National Science Foundation (US), the Particle Physics and Astronomy Research Council (UK), the National Research Council (Canada), CONICYT (Chile), the Australian Research Council (Australia), CNPq (Brazil), and CONICET (Argentina).

\section{REFERENCES}

Aitken, D. K., Briggs, G., Bailey, J. A., Roche, P. F., \& Hough, J. H. 1984, Nature, 310,660

Antonucci, R. R. J., \& Miller, J. S. 1985, ApJ, 297, 621

Bailey, J., Axon, D. J., Hough, J. H., Ward, M. J., McLean, I., \& Heathcote, S. R. 1988, MNRAS, 234, 899

Berry, D. S., \& Gledhill, T. M. 2003, Starlink User Notes, http://star-www.rl.ac.uk Catchpole, R. M., \& Boksenberg, A. 1997, Ap\&SS, 248, 79

Davies, R., Genzel, R., Tacconi, L., Mueller Sanchez, F., \& Sternberg, A. 2006, preprint (astro-ph/0612009)

Elitzur, M., \& Shlosman, I. 2006, ApJ, 648, L101

Galliano, E., Alloin, D., Granato, G. L., \& Villar-Martín, M. 2003, A\&A, 412, 615

Galliano, E., Pantin, E., Alloin, D., \& Lagage, P. O. 2005, MNRAS, 363, L1

Gallimore, J. F., Henkel, C., Baum, S. A., Glass, I. S., Claussen, M. J., Prieto, M. A., \& Von Kap-herr, A. 2001, ApJ, 556, 694

Glasse, A. C. H, Atad-Ettedgui, E. I., \& Harris, J. W. 1997, Proc. SPIE, 2871, 1197

Greenhill, L. J., \& Gwinn, C. R. 1997, Ap\&SS, 248, 261

Jackson, J. M., Paglione, T. A. D., Ishizuki, S., \& Nguyen-Q-Rieu. 1993, ApJ, 418, L13

Jaffe, W., et al. 2004, Nature, 429, 47

Lazarian, A. 2003, J. Quant. Spectrosc. Radiat. Transfer, 79, 881

Lumsden, S. L., Moore, T. J. T., Smith, C., Fujiyoshi, T., Bland-Hawthorn, J., \& Ward, M. J. 1999, MNRAS, 303, 209

Mason, R. E., Geballe, T. R., Packham, C., Levenson, N. A., Elitzur, M., Fisher, R. S., \& Perlman, E. 2006, ApJ, 640, 612

Nenkova, M., Ivezić, Ž., \& Elitzur, M. 2002, ApJ, 570, L9

Ogle, P. M., Brookings, T., Canizares, C. R., Lee, J. C., \& Marshall, H. L. 2003, A\&A, 402, 849
Packham, C., Radomski, J. T., Roche, P. F., Aitken, D. K., Perlman, E., AlonsoHerrero, A., Colina, L., \& Telesco, C. M. 2005, ApJ, 618, L17

Packham, C., Young, S., Hough, J. H., Axon, D. J., \& Bailey, J. A. 1997, MNRAS, 288, 375

Planesas, P., Scoville, N., \& Myers, S. T. 1991, ApJ, 369, 364

Radomski, J. T., et al. 2006, BAAS, 209, 149.11

Rhee, J. H., \& Larkin, J. E. 2006, ApJ, 640, 625

Roche, P. F., Packham, C., Aitken, D. K., \& Mason, R. E. 2007, MNRAS, 375, 99

Roche, P. F., Packham, C., Telesco, C. M., Radomski, J. T., Alonso-Herrero, A., Aitken, D. K., Colina, L., \& Perlman, E. 2006, MNRAS, 367, 1689

Roche, P. F., Whitmore, B., Aitken, D. K., \& Phillips, M. M. 1984, MNRAS, 207, 35

Schinnerer, E., Eckart, A., Tacconi, L. J., Genzel, R., \& Downes, D. 2000, ApJ, 533, 850

Siebenmorgen, R., Krügel, E., \& Spoon, H. W. W. 2004, A\&A, 414, 123

Simpson, J. P., Colgan, S. W. J., Erickson, E. F., Hines, D. C., Schultz, A. S. B., \& Trammell, S. R. 2002, ApJ, 574, 95

Smith, C. H., Wright, C. M., Aitken, D. K., Roche, P. F., \& Hough, J. H. 2000, MNRAS, 312, 327

Watanabe, M., Nagata, T., Sato, S., Nakaya, H., \& Hough, J. H. 2003, ApJ, 591,714

Weigelt, G., Wittkowski, M., Balega, Y. Y., Beckert, T., Duschl, W. J., Hofmann, K.-H., Men'shchikov, A. B., \& Schertl, D. 2004, A\&A, 425, 77

Young, S., Hough, J. H., Axon, D. J., Bailey, J. A., \& Ward, M. J. 1995, MNRAS, 272, 513

Young, S., Packham, C., Hough, J. H., \& Efstathiou, A. 1996, MNRAS, 283, L1 